\title{
Correction to: Is the ghost of brown tumor back again? Features of hypercalcaemic primary hyperparathyroidism we must not forget
}

\author{
Irene Gagliardi $\mathbb{D}^{1} \cdot{\text { Alessandro } \text { Mella }^{2} \cdot \text { Paola Franceschetti }}^{1,2} \cdot$ Maria Rosaria Ambrosio $\mathbb{D}^{1}$. \\ Maria Chiara Zatelli $\mathbb{i D}^{1,2}$
}

Published online: 30 October 2021

(c) Springer Science+Business Media, LLC, part of Springer Nature 2021

Correction to: Endocrine https://doi.org/10.1007/s12020-02102896-7, Published online 09 October 2021

In this article the following author name (Paola Franceschetti) was missing from the author list.
This has been corrected in this erratum. The original article has been corrected.

Maria Chiara Zatelli

ztlmch@unife.it

1 Section of Endocrinology, Geriatrics and Internal Medicine, Department of Medical Sciences, University of Ferrara, Ferrara, Italy

2 Unit of Endocrinology and Metabolic Diseases - Oncology and Specialty Medicines Department, Azienda Ospedaliero Universitaria di Ferrara, Ferrara, Italy 